\title{
SOGRO (Superconducting Omni-directional Gravitational Radiation Observatory)
}

\author{
Ho Jung Paik ${ }^{1, *}$ \\ ${ }^{1}$ Department of Physics, University of Maryland, College Park, MD 20742, U.S.A.
}

\begin{abstract}
Detection of gravitational waves (GWs) from merging binary black holes (BHs) by Advanced LIGO has ushered in the new era of GW astronomy. Many conceivable sources such as intermediate-mass BH binaries and white dwarf binaries, as well as stellar-mass $\mathrm{BH}$ inspirals, would emit GWs be low $10 \mathrm{~Hz}$. It is highly desirable to open a new window for GW astronomy in the infrasound frequency band. A low-frequency tensor detector could be constructed by combining six magnetically levitated superconducting test masses. Such a detector would be equally sensitive to GWs coming from anywhere in the sky, and would be capable of resolving the source direction and wave polarization. I will present the design concept of a new terrestrial GW detector, named SOGRO, which could reach a strain sensitivity of $10^{-19}-10^{-21} \mathrm{~Hz}^{-1 / 2}$ at $0.1-10 \mathrm{~Hz}$. Seismic and Newtonian gravity noises are serious obstacles in constructing terrestrial GW detectors at frequencies below $10 \mathrm{~Hz}$. I will explain how these noises are rejected in SOGRO. I will also report the progress made in designing the platform and modelling its thermal noise.
\end{abstract}

\section{Introduction}

Detection of gravitational waves (GWs) from merging binary black holes (BHs) by the two Advanced LIGO (aLIGO) detectors has ushered in the new era of GW astronomy [1-3]. Now, the Advanced Virgo (AdV) detector has joined the search for GWs. These laser interferometers cover a frequency band from $\sim 10 \mathrm{~Hz}$ to several kilohertz. The LISA mission, selected by ESA for launch in 2034 [4], would observe GWs over a low-frequency band from $0.1 \mathrm{mHz}$ to $0.1 \mathrm{~Hz}$. This would still leave the frequency band $f=0.1-10 \mathrm{~Hz}$ uncovered, where interesting astrophysical sources such as intermediate-mass black hole (IMBH) and white dwarf(WD) binaries could be observed.

Ando et al. [5] proposed a space interferometer, DECIGO, to cover the missing midfrequency band. Harms et al. [6] looked into the poss ibility of constructing a mid-frequency terrestrial detector by using an atom-laser hybrid interferometer, a differential torsion-bar detector (TOBA), and a conventional laser interferometer. Paik et al. [7] proposed a terrestrial tensor GW detector, SOGRO (Superconducting Omni-directional Gravitational Radiation Observatory), consisting of six levitated superconducting test masses, to cover the same frequency band. Such a detector would be equally sensitive to GWs coming from

\footnotetext{
Corresponding author: hpaik@umd.edu
} 
anywhere in the sky, and would be capable of resolving the source direction and wave polarization. In this paper, I discuss the design concept of SOGRO and report the recent progress made in designing the platformand modelling its thermalnoise.

Unlike the other detectors, SOGRO requires a large-scale cryogenic operation since the test masses must be levitated from a rigid platform and the entire platform needs to be cooled to $4.2 \mathrm{~K}$ or below to reduce its thermal noise. Therefore, our initial research effort was directed to conceptual design of the platformand modelling theplatformnoise.

\section{Design Concept and Operating Principle}

The potential of using two widely separated test masses coupled by an electrical circuit to detect GWs was realized in the 1970s by Wagoner et al. [8]. SOGRO is a 3D extension of such a long-baseline resonant-mass detector. Figure 1 shows a perspective view of SOGRO. Six superconducting test masses, each with three linear degrees of freedom, are levitated over three orthogonal mounting tubes. The test masses are made of niobium $(\mathrm{Nb})$ in the shape of a rectangular shell. Superconducting levitation/alignment coils and sensing capacitors (not shown) are located in the gap between the test masses and the mounting tubes, as well as on the outer surfaces of the test masses. The along-axis motions of the two test masses on each coordinate axis are differenced to measure a diagonal component of the wave while the cross-axis (rotational) motions of the four test masses on each coordinate plane are differenced to meas ure an off-diagonal component of the wave:

$$
\begin{gathered}
h_{i i}(t)=\frac{2}{L}\left[x_{+i i}(t)-x_{-i i}(t)\right], \\
h_{i j}(t)=\frac{1}{L}\left\{\left[x_{+i j}(t)-x_{-i j}(t)\right]-\left[x_{-j i}(t)-x_{+j i}(t)\right]\right\}, i \neq j,
\end{gathered}
$$

where $x_{ \pm j}(\mathrm{t})$ is displacement of the test mass on the $\pm i$ axis along the $j$ axis and $L$ is the separation between the test mas ses on each axis.

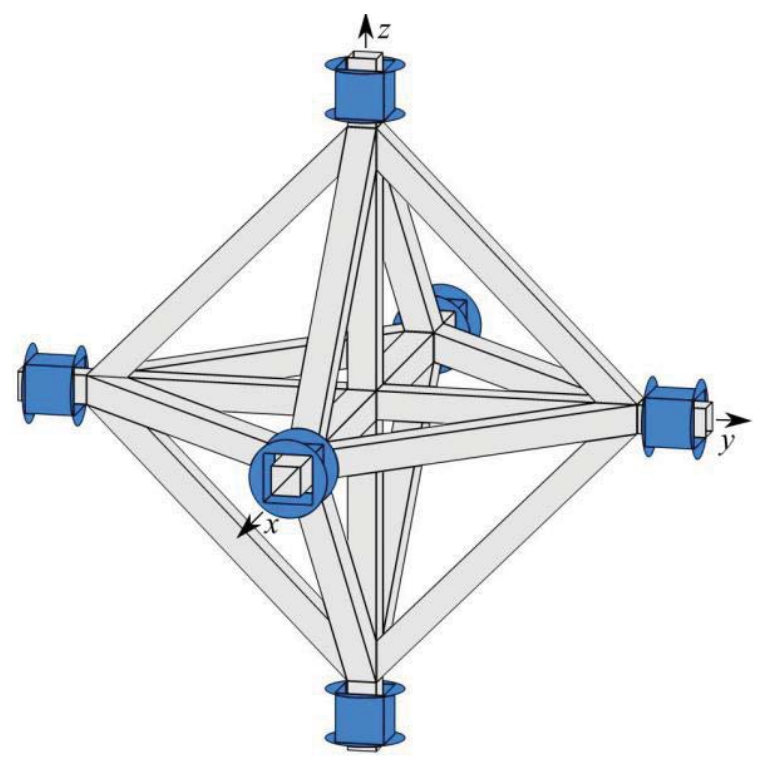

Fig. 1. Perspective view of SOGRO. Six magnetically levitated superconducting test masses are combined to measure all six components of the curvature tensor. 


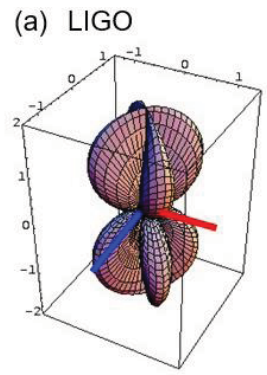

$\times$ polarization

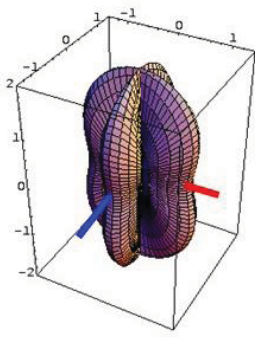

+ polarization

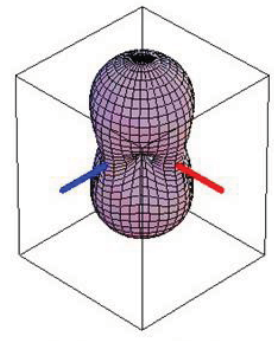

RMS sensitivity

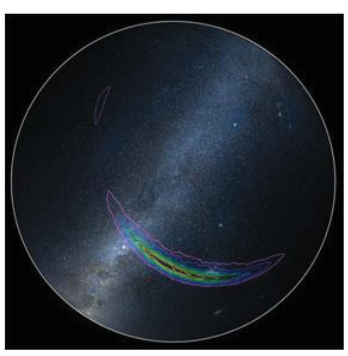

Sky location

(b) SOGRO

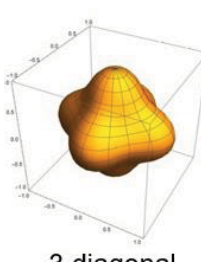

3 diagonal
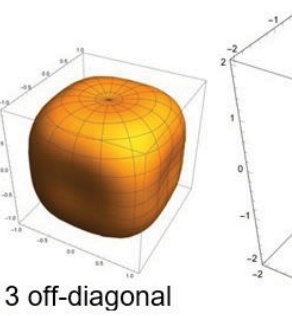

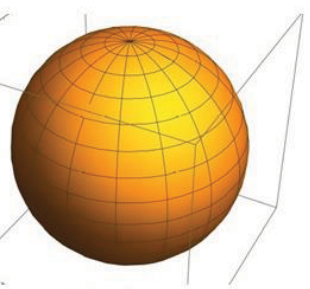

Full tensor

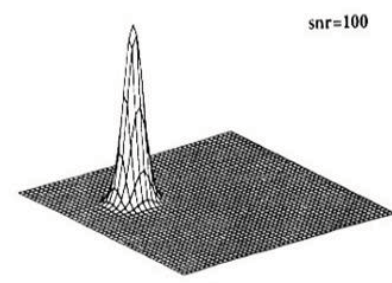

Sky location

Fig. 2. Antenna pattern of SOGRO compared with that of LIGO. Being a full-tensor detector, SOGRO has full-sky coverage with equal sensitivity to waves coming from any direction with any polarization and a single SOGRO can locate the source in the sky.

Figure 2 compares the antenna pattern of SOGRO with that of LIGO. Being a fulltensor detector, SOGRO has equal sensitivity to waves coming from any direction with any polarization. A single SOGRO can locate the source in the sky, modulo angle $\pi$, without necessitating triangulation.

The operating principle of SOGRO follows closely that of the tens or superconducting gravity gradiometer (SGG) under development at the University of Maryland [9]. Since test mass motion is measured with respect to the sensing circuit elements mounted on the platform, SOGRO requires a rigid platform with its mode frequencies above the signal bandwidth. To alleviate excessive demand on cryogenics, the platform must not be too heavy yet sufficiently rigid.

Each test mass weighs 5 tons and has a large enough surface area to be levitated against earth's gravity with a magnetic field of $0.10 \mathrm{~T}$, well below $0.14 \mathrm{~T}$, the first critical field $\left(H_{c 1}\right)$ of high-purity $\mathrm{Nb}$ at $4.2 \mathrm{~K}$. Magnetic levitation allows the test masses to have horizontal differential-mode $(\mathrm{DM})$ resonance frequencies of $\sim 0.01 \mathrm{~Hz}$. In the vertical direction, the DM frequencies become $\sim 1 \mathrm{~Hz}$ due to the stiffness aris ing from nonlinearities in the levitation coils. This implies that the $h_{x z}, h_{y z}$ and $h_{z z}$ channels, which utilize vertical accelerometers, will be noisier. Fortunately, there is a way to detect all the tensor components by combining only the horizontal motions of the test masses. Since the GW tensor is traceless, $h_{z z}$ can be obtained from $h_{z z}=-\left(h_{x x}+h_{y y}\right)$ without necessitating measurement of vertical accelerations. According to Eq. (2), $h_{x z}$ is obtained by combining the relative $z$ displacement of the two test masses on the $x$ axis with the relative $x$ displacement of the two test mas ses on the $z$ axis:

$$
h_{x z}=\frac{1}{L}\left[\left(z_{x 2}-z_{x 1}\right)+\left(x_{z 2}-x_{z 1}\right)\right]
$$

Since the platform is completely isolated from the ground tilt (see Sec. 5), $z_{x 2}-z_{x 1}$ and $x_{z 2}-$ $x_{z 1}$ are not contaminated by any common-mode (CM) angular acceleration. Further, $h_{x z}$ 
drives the two arms symmetrically: $z_{x 2}-z_{x 1}=x_{z 2}-x_{z 1}$. The same holds for $h_{y z}$. Therefore, thes e tensor components can als o be obtained fromhorizontal dis placements only.

$$
h_{x z}=\frac{2}{L}\left(x_{z 2}-x_{z 1}\right), \quad h_{y z}=\frac{2}{L}\left(y_{z 2}-y_{z 1}\right) .
$$

The tiny mechanical energy that a GW deposits into the antenna must be converted into an electrical signal and coupled to a nearly quantum-limited SQUID (Superconducting Quantum Interference Device). Unfortunately, nearly quantum-limited dc SQUIDs have a $1 /$ f noise below several $\mathrm{kHz}$. We need to use an active transducer to upconvert the signal frequency to above the $1 / \mathrm{f}$ noise comer frequency. For SOGRO, we propose to employ a superconducting capacitance bridge transducer coupled to a dc SQUID. A similar trans ducer was developed by Cinquegrana et al. [10] for res onant-mass GW detectors.

Figure 3 shows the transducer for a diagonal-component channel. $C_{i j}(t)$ is a $\mathrm{Nb}$ capacitor plate located near the $j$-th face of the $i$-th test mass. The bridge output is coupled to a nearly quantum-limited dc SQUID through an impedance-matching superconducting transformer. The sensing capacitances are modulated by GWs at $\omega$ and the bridge is driven at $\omega_{p}>\omega$, well above the 1/f noise comer frequency of the SQUID. An active transducer introduces a new noise source, the oscillator noise. Although a bridge configuration is employed to null the carrier, the sideband components contained in the carrier can become a dominant noise source, if the bridge balance is not sufficiently precise. In our design, the carrier signal is precisely balanced by applying feedback forces to the test masses, eliminating the oscillator noise at the output.

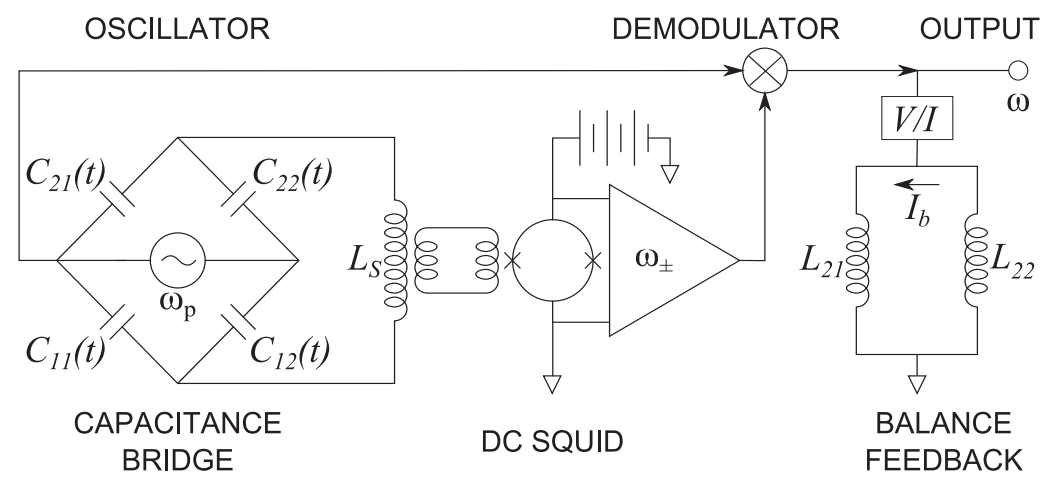

Fig. 3. Capacitance-bridge transducer coupled to a nearly quantum-limited dc SQUID. The bridge is driven at the resonance frequency of the capacitance bridge tuned with the SQUID input coil inductance. Feedback is applied to keep the bridge precisely balanced.

\section{Platform Design and Noise Analysis}

An efficient design of the platform and the cryos tat is of utmost importance for SOGRO. We have carried out FEM analys is for various platform design options with $L=30,50$ and $100 \mathrm{~m}$. Our ANSYS analys is shows that, by building the 3D cross with aluminum (Al) tubes triangulated by Al tube struts and fortified by additional braces at the points where thes e tubes meet, a 50-m platform can be constructed with all the mode frequencies above $10 \mathrm{~Hz}$ and a total mass of $\sim 220$ tons (see Fig. 4). To increase their intemal mode frequencies, the test masses are made compact by using folded $\mathrm{Nb}$ shells and are levitated inside the orthog onal Al tubes near the six vertices of the platform. This gives an additional advantage of being able to shrink the size of the cryostat. 


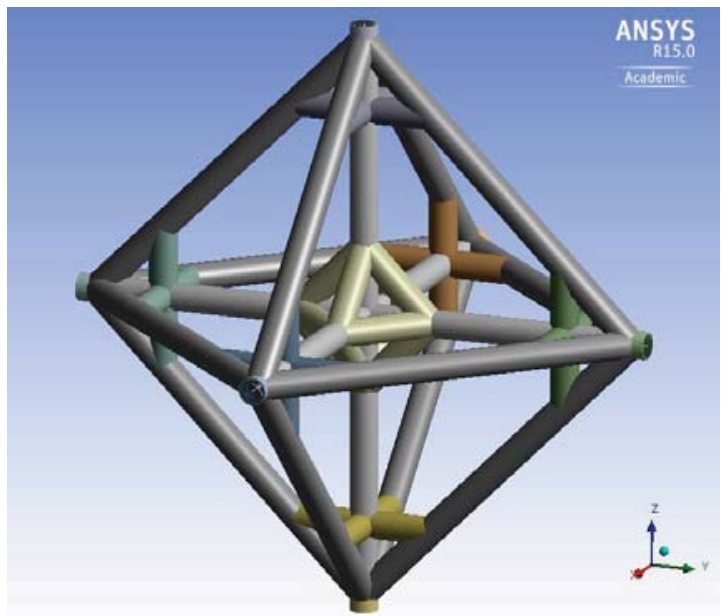

Fig. 4. 50-m platform constructed with $2.5-\mathrm{m}$ diameter Al tube with 1-cm thick wall, triangulated with diagonal $\mathrm{Al}$ tube struts and fortified by additional braces.

For the platform noise, we assume an internal damping model, which modifies Hooke's law as $F=-k(1+i \phi) x$, where $\phi$ is the loss angle. The thermal noise displacement power spectral density (PSD) is given [11] by

$$
S_{\xi}(f)=\frac{4 k_{B} T \omega_{p l}^{2}}{M_{p l} \omega Q_{p l}\left[\left(\omega_{p l}^{2}-\omega^{2}\right)^{2}+\left(\omega_{p l}^{2} / Q_{p l}\right)^{2}\right]},
$$

where $\omega_{p l}$ is the resonance frequency of the mode under consideration, $M_{p l}$ is the effective mass of the platform, which depends on where the displacement $\xi$ is measured, and $Q_{p l}$ is the quality factor of the mode. To compute $S_{\xi}(f)$, a pre-stressed modal analysis was performed using ANSYS finite element software.

Figure 5 shows the platform thermal noise coming from dominant $x x$ longitudinal platform modes in (a), and dominant $x y$ scissor modes of the platform in (b), at $T=4.2 \mathrm{~K}$ assuming $Q_{p l}=10^{7}$. All the mode frequencies are above $10 \mathrm{~Hz}$ except for one scissor mode. The noise summed over six dominant modes gives a level lower than or comparable to the intrins ic detector noise of Advanced SOGRO (aSOGRO) for both $h_{x x}$ and $h_{x y}$ channels (see Fig. 6). (Other modes make negligible contributions.) For initial SOGRO, $Q_{p l}=10^{6}$ would be sufficient to keep the platform thermal noise below the intrinsic detector noise level.

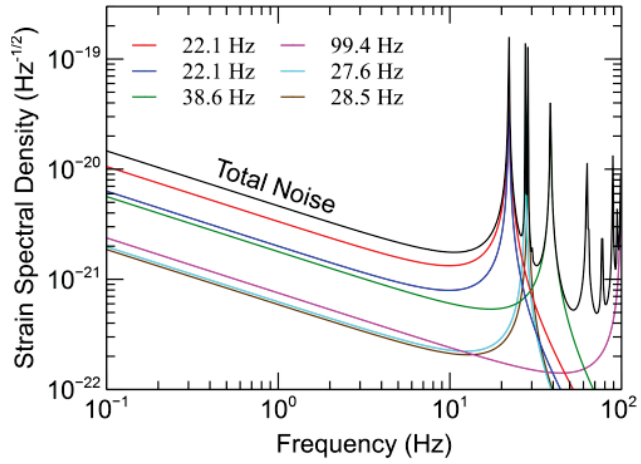

(a) Longitudinal modes

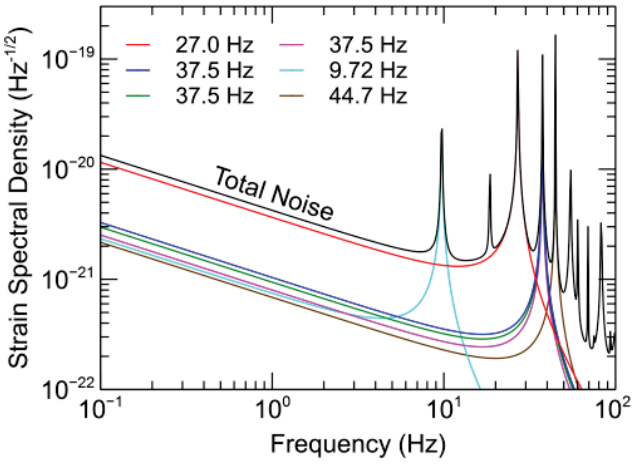

(b) Scissor modes

Fig. 5. Plat form thermal noise coming from (a) dominant longitudinal modes and (b) dominant scissor modes at $T=4.2 \mathrm{~K}$ assuming $Q_{p l}=10^{7}$. 
Obtaining $Q_{p l}=10^{7}$ in a composite structure will be extremely challenging even at cryogenic temperatures. However, we expect that the Q requirement can be relaxed by at least an order of magnitude as we optimize the design of the platform. $M_{p l}$ for a particular mode becomes infinity at its nodes, resulting in $S_{\xi}(f)=0$. With the additional degrees of freedom introduced by the corner braces, one could adjust the shapes and positions of these braces to move the nodal points of dominant modes nearer to the test mass locations. An indepth en gineering study is required to optimize the platform design.

\section{Detector Sensitivity}

The detector sensitivity is limited by the intrinsic detector noise (antenna thermal plus amplifier noise) and the platform thermal noise. Seismic and Newtonian gravity noises (NN) are important extemal noise sources which can also limit the detector resolution. In this section, I will discuss the intrinsic detector noise and the platform noise. Seismic noise is olation and NN mitigation will be described in Sec. 5 .

The detector noise PSD of an off-diagonal-component GW detector with an active trans ducer can be shown [7] to be

$$
S_{h}(f)=\frac{16}{M L^{2} \omega^{4}}\left[\frac{k_{B} T \omega_{D}}{Q_{D}}+\frac{\left|\omega^{2}-\omega_{D}^{2}\right|}{\omega_{p}}\left(1+\frac{1}{\beta^{2}}\right)^{1 / 2} k_{B} T_{N}\right]
$$

where $M$ and $L$ are the mass of each test mass and the arm-length of the detector, $T$ is the temperature; $\omega_{D}$ and $Q_{D}$ are the DM (angular) resonance frequency and Q; and $\beta$ and $T_{N}$ are the energy coupling constant of the transducer and the noise temperature of the SQUID, respectively. One obvious way of increas ing the sensitivity is enlarging $M$ and $L$. However, it will require cooling a larger mass. In this paper, we consider a detector with $M=5 \times 10^{3}$ $\mathrm{kg}$ and $L=50 \mathrm{~m}$, which brings the total mass including the platform to $\sim 250$ tons. In an active transducer, it is possible to achieve $\beta>1$. For our tuned capacitance-bridge trans ducer, we have

$$
\beta=\frac{2 C E_{p}^{2} Q_{p}}{M\left|\omega^{2}-\omega_{D}^{2}\right|} \frac{1}{\sqrt{1+\left(2 Q_{p} \omega / \omega_{p}\right)^{2}}},
$$

where $C$ is the equilibrium capacitance of each sens ing capacitor, $E_{p}$ is the amplitude of the driving electric field at $\omega_{p}$, and $Q_{p}$ is the electricalQ.

Table 1 shows the proposed detector parameter values and detector noise for SOGRO and aSOGRO. Both detectors have an arm-length of $50 \mathrm{~m}$ and test masses weighing 5 tons each. The amplifier noise number is defined by $n \equiv k_{B} T_{N} \hbar \omega_{p}$. SOGRO is cooled to $4.2 \mathrm{~K}$ by liquid helium and aSOGRO to $0.1 \mathrm{~K}$ by using $\mathrm{He}^{3} / \mathrm{He}^{4}$ dilution refrigerators. White noise levels of $10 \hbar$ have been demonstrated at $0.1 \mathrm{~K}$ by us ing a two-stage dc SQUID, in which a commercial sensor was used as the preamplifier for the first SQUID [12]. We set $20 \hbar$ and $5 \hbar$ as the target SQUID noise for SOGRO and aSOGRO, respectively. SOGRO has very stringent $\mathrm{Q}$ requirement. Although no fundamental limit is known for the $\mathrm{Q}$ of levitated superconducting masses, parasitic forces due to trapped magnetic flux and patch fields will cause damping in the transducer. To minimize such parasitic forces, the test masses will be constructed with high-purity $\mathrm{Nb}$ and with the surface highly polished.

In Fig. 6, we plot the expected strain sensitivities of SOGRO and aSOGRO, as well as those of aLIGO, AdV and eLISA. The NN due to the Rayleigh waves and infrasound waves before mitigation are also plotted $[6,13]$. The SOGRO detector noise is dominated by the 
Table 1. Proposed detector parameters and detector noise of SOGRO.

\begin{tabular}{|l|c|c|l|}
\hline Parameter & SOGRO & aSOGRO & Method employed (/aSOGRO) \\
\hline Each test mass $M$ & $5 \mathrm{ton}$ & $5 \mathrm{ton}$ & Nb shell \\
\hline Arm-length $L$ & $50 \mathrm{~m}$ & $50 \mathrm{~m}$ & Over “rigid" platform \\
\hline Antenna temp $T$ & $4.2 \mathrm{~K}$ & $0.1 \mathrm{~K}$ & LHe $/ \mathrm{He}^{3}-\mathrm{He}^{4}$ dilution refrigerator \\
\hline Platform temp $T_{D l}$ & $4.2 \mathrm{~K}$ & $4.2 \mathrm{~K}$ & Large-scale cryogenics \\
\hline Platform Q factor $Q_{D l}$ & $10^{\supset}$ & $10^{\circ}$ & Al tube construction \\
\hline DM frequency $f_{D}$ & $0.01 \mathrm{~Hz}$ & $0.01 \mathrm{~Hz}$ & Magnet ic levitation (horizontal) \\
\hline DM quality factor $Q_{D}$ & $10^{\prime}$ & $10^{\gamma}$ & Surface polished pure Nb \\
\hline Pump frequency $f_{D}$ & $50 \mathrm{kHz}$ & $50 \mathrm{kHz}$ & Tuned capacitor bridge transducer \\
\hline Amplifier noise no. $n$ & 20 & 5 & 2-stage dc SQUID cooled to $0.1 \mathrm{~K}$ \\
\hline Detector noise $S_{h}{ }^{1 / 2}(f)$ & $2.9 \times 10^{-20} \mathrm{~Hz}^{-1 / 2}$ & $5.6 \times 10^{-21} \mathrm{~Hz}^{-1 / 2}$ & Computed at 1 Hz \\
\hline
\end{tabular}

antenna thermal nois e below $0.2 \mathrm{~Hz}$, by the amplifier noise at $0.2-1 \mathrm{~Hz}$, and by the platform thermal noise above $1 \mathrm{~Hz}$. aSOGRO has potential sensitivity $\leq 10^{-20} \mathrm{~Hz}^{-1 / 2}$ at $0.5-10 \mathrm{~Hz}$. To reach the detector noise limit, the NN must be mitigated by up to $70 \mathrm{~dB}$. The sensitivity of aSOGRO surpasses those of terrestrial and space laser interferometers between 0.1 and 10 $\mathrm{Hz}$ and fills the frequency gap between them. In addition to detecting the IMBH and WD binaries, aSOGRO will be sensitive enough to be able to detect stellar mass BH binaries like GW 150914 and warn the las er interferometers days before merger.

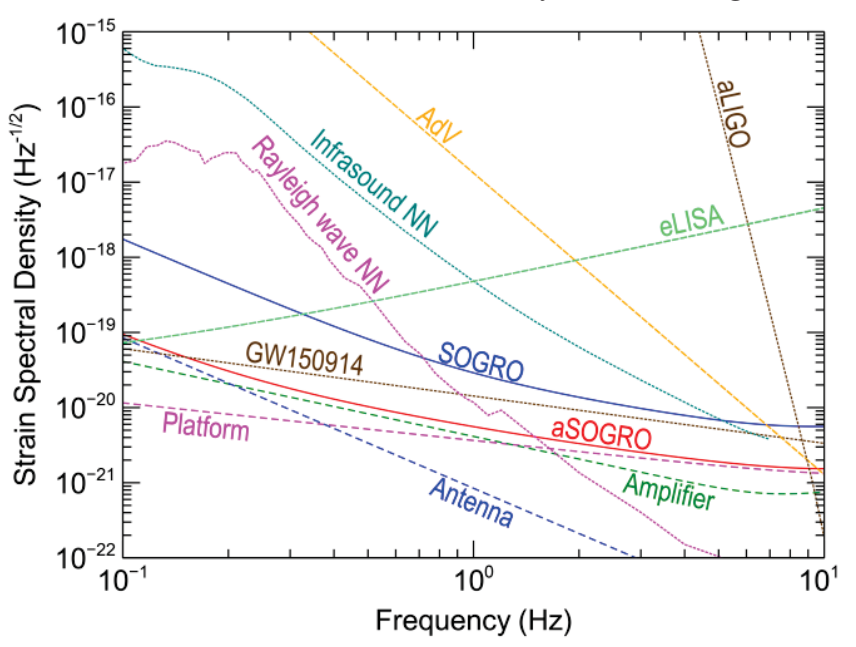

Fig. 6. Expected strain sensitivities of SOGRO and a SOGRO as well as those of aLIGO, AdV and eLISA. a SOGRO will be sensitive enough to be able to detect stellar mass BH binaries like GW 150914 and to warn the laser interferometers days before merger.

\section{Seismic and Newtonian Noise}

We propose to construct SOGRO underground at a depth $\geq 500 \mathrm{~m}$. The seis mic noise level of an underground lab at $0.5-10 \mathrm{~Hz}$ is $\leq 5 \times 10^{-8} \mathrm{~m} \mathrm{~s}^{-2} \mathrm{~Hz}^{-1 / 2}$ [14], 10 orders of magnitude above the target sensitivity of SOGRO. Vibration isolation at such low frequencies is extremely difficult. The CM rejection techniques demonstrated with SGGs [15], which produced a stable CM rejection level of three parts in $10^{8}$, will be applied to SOGRO and improved to one part in $10^{9}$. The highly polished, high-purity $\mathrm{Nb}$ surfaces will allow for 
exceptional stability of the balance and error rejection. This will reduce the vibration is olation requirement to $20 \mathrm{~dB}$.

The entire platform will be suspended as a pendulum from outside the cryostat with the suspension cable attached to just above its center of mass. The resulting $25-\mathrm{m}$ pendulum will have its pendulum frequency at $0.1 \mathrm{~Hz}$ and a torsional mode frequency $\leq 1 \mathrm{mHz}$. This pendulum suspension will completely decouple the platform from the ground tilt, and provide passive isolation of $40 \mathrm{~dB}$ to horizontal accelerations and $120 \mathrm{~dB}$ to angular accelerations at $1 \mathrm{~Hz}$, and much more at $10 \mathrm{~Hz}$. Combined with the overall $\mathrm{CM}$ rejection of $180 \mathrm{~dB}$, the seis mic noise could be reduced to below the intrins ic detector noise level in all degrees of freedom except for the vertical. For the vertical direction, 20-dB isolation must be provided by combining passive and active isolation.

The NN generated by moving local masses poses a formidable challenge to approaching the detector noise limit at $f \leq 1 \mathrm{~Hz}$. At low frequencies, the $\mathrm{NN}$ is dominated by Rayleigh waves and infrasound waves. Both the Rayleigh and infrasound waves are exponentially suppressed with depth. For the Rayleigh waves $\left(c_{R}=3.5 \mathrm{~km} \mathrm{~s}^{-1}\right)$, there is hardly any attenuation at a $500-\mathrm{m}$ depth. However, for the infrasound $\left(c_{I S}=330 \mathrm{~m} \mathrm{~s}^{-1}\right)$, the $\mathrm{NN}$ is attenuated by up to a factor 6 at $0.2 \mathrm{~Hz}$ and to $<10^{-8}$ at $1 \mathrm{~Hz}$ at the same depth. At $0.2 \mathrm{~Hz}$, where micros eis ms peak, the NN must be rejected by another three orders of magnitude to reach the intrinsic noise limit. SOGRO is able to meet this challenge by utilizing the full tensor measurement and simultaneous measurement of environmental density fluctuations. A detailed analysis has been published els ewhere [16]. I summarize our findings here.

Consider a GW coming from $(\theta, \phi)$ direction in the presence of Rayleigh and in frasound waves, where $\theta$ and $\phi$ are the polar (with respect to zenith) and the azimuthal angle, res pectively. The full strain tensor in the GW coordinates has the form:

$$
h^{\prime}(\omega)=\left(\begin{array}{ccc}
h_{+}(\omega)+h_{N 11}^{\prime}(\omega) & h_{\times}(\omega)+h_{N 12}^{\prime}(\omega) & h^{\prime}{ }_{N 13}(\omega) \\
h_{\times}(\omega)+h_{N 12}^{\prime}(\omega) & -h_{+}(\omega)+h_{N 22}^{\prime}(\omega) & h_{N 23}^{\prime}(\omega) \\
h_{N 13}^{\prime}(\omega) & h_{N 23}^{\prime}(\omega) & h_{N 33}^{\prime}(\omega)
\end{array}\right),
$$

where $h_{+}(\omega)$ and $h_{\times}(\omega)$ are the two polarization components of the GW, and $h^{{ }^{\prime}}{ }_{N i j}(\omega)$ are the $\mathrm{NN}$ components. Due to the transverse nature of the GW, $h{ }_{13}, h_{23}, h_{33}$, and $h{ }^{\prime}{ }_{11}+h^{\prime}{ }_{22}$ contain only the NN components. Since a Rayleigh wave has three unknowns (amplitude, phase, incident angle $\phi$ ) and an infrasound wave has four (amplitude, phase, incident angles $\theta$, $\phi$ ) while eight $\mathrm{NN}$ variables (amplitude and phase of $h_{N 13}^{\prime}, \underline{h}_{\underline{2} 23}^{\prime}, h_{N 33}^{\prime}, h_{N 11}^{\prime}+h_{N 22}^{\prime}$ ) are measured, SOGRO can determine up to a combination of any two waves.

In the general case where more than two waves are incident simultaneously, SOGRO must be supplemented with external seismometers and microphones and the NN must be removed by using a correlation method. Figure 7(a) shows the residual NN achieved for Rayleigh waves in the absence of infrasound waves by using $h_{13}^{\prime}, h_{23}^{\prime}, h_{33}^{\prime}$ and $a_{z}(\mathrm{CM})$, plus seven seis mometers with SNR of $10^{3}$ at the radius of $5 \mathrm{~km}$ as the input of the Wiener filter. The $\mathrm{NN}$ has been removed to $\sim 10^{-3}$ with environmental sensors (s eis mometers) alone. The local channels of SOGRO improve the noise significantly only near $\theta=\pi / 2$.

Figure 7(b) is the residual NN achieved for infrasound waves in the absence of Rayleigh waves by using $h_{13}^{\prime}, h_{23}^{\prime}, h_{33}^{\prime}$ and 15 microphones of SNR of $10^{4}$, one at the detector, seven each at radii of $600 \mathrm{~m}$ and $1 \mathrm{~km}$ around the detector. With the environmental sensors (microphones) alone, the NN cannot be mitigated except at $\theta=0, \pi / 2$, and $\pi$. This is because the infrasound waves come from a half-space and the microphones deployed over a surface are insufficient to measure the effect of 3D density variations of the atmosphere. The vertical strain component $h_{33}^{\prime}$ of SOGRO largely makes up for this deficiency. With the aid of the local strain channels, the NNhas been rejected to $10^{-3}$ for all $\theta$. 


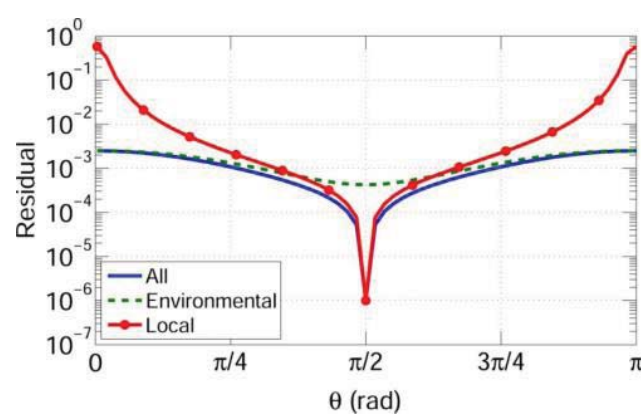

(a) Residual Rayleigh wave NN

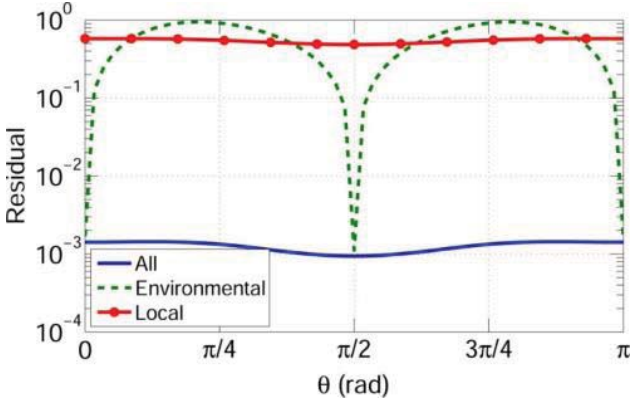

(b) Residual infrasound wave NN

Fig. 7. (a) Residual $\mathrm{NN}$ achieved for Rayleigh waves by using $h^{\prime}{ }_{13}, h_{23}^{\prime}, h_{33}^{\prime}$ and $a_{z}$, plus seven seismometers with SNR $=10^{3}$ at $R=5 \mathrm{~km}$. (b) Residual NN achieved for infrasound by using $h^{\prime}{ }_{13}$, $h^{\prime}{ }_{23}, h^{\prime}{ }_{33}$ and 15 microphones of SNR $=10^{4}$, one at the detector, seven each at $R=600 \mathrm{~m}$ and $1 \mathrm{~km}$

With both waves present, the resulting mixed NN cannot be removed by using these methods. We propose to use an array of external seismometers to first remove the Rayleigh waves independently of the infras ound to one part in $10^{3}$, and then combine the cleaned-up $h_{13}^{\prime}, h_{23}^{\prime}, h^{\prime}{ }_{33}$ and microphone signals to remove the remaining $\mathrm{NN}$ due to the infrasound to one part in $10^{3}$. This will satis fy the rejection requirement for both types of NN for SOGRO as well as aSOGRO.

\section{Conclusions}

A wideband terrestrial tensor GW detector with a strain sensitivity of $10^{-19}-10^{-21} \mathrm{~Hz}^{-1 / 2}$ for the frequency band of $0.1-10 \mathrm{~Hz}$ could be constructed by using six widely separated, magnetically levitated superconducting test masses. Being a full-tensor detector, a single SOGRO antenna would be capable of determining the source direction and wave polarization for signals with sufficient SNR. The tensor outputs could be combined to better reject the $\mathrm{NN}$ caused by the ground motion and atmospheric density fluctuations. This way, NN mitigation can be achieved at the same level as in conventional GW detectors independentlyof the GW direction ofpropagation [16].

A major technical challenge is the construction of a large $(50 \mathrm{~m}, 200+$ ton $)$, rigid enough $(\geq 10 \mathrm{~Hz}), 3 \mathrm{D}$ platform with high $\mathrm{Q}\left(\geq 10^{6}\right)$ that can be cooled to the liquid helium temperature. Detailed engineering studies need to be performed to find a platform design which reduces the weight while providing sufficient rigidity. Another challenge is obtaining the required high $\mathrm{Q}\left(\sim 10^{8}\right)$ in the levitated superconducting test masses. A dedicated experimental research effort will be required to achieve such a high $\mathrm{Q}$.

Although very challenging, SOGRO has a number of attractive features and deserves a serious consideration as an option for future low-frequency terrestrial GW detectors. SOGRO will be the first tensor detector that can perform genuine full-sky search for GW events. With SOGRO, one could set a new limit for GW flux at $f=0.1-10 \mathrm{~Hz}$. Binaries composed of two IMBHs of $10^{4} M_{\odot}$ at the distance of a few billion light years or binaries composed of two WDs within the Local Group could be detected.

A sub-Hz high-precision GW detector has other useful applications. A recent study [17] shows that a gravity strain sens or with $10^{-20} \mathrm{~Hz}^{-1 / 2}$ sensitivity at $0.1 \mathrm{~Hz}$ would detect an early gravity perturbation induced by an earthquake of magnitude $M>6$ with SNR $>5$ at epicentral distances up to $500 \mathrm{~km}$. The prompt detection of earthquakes would enable an early waming to be issued before destructive seismic waves reach populated areas. Another potential application of SOGRO is mitigation of the $\mathrm{NN}$ for future third-generation $\mathrm{GW}$ detectors such as post advanced LIGO interferometers [18] and ET [19]. A set of shorter 
arm-length $(\sim 5 \mathrm{~m})$ SOGROs located in close proximity to the interferometer test masses could detect the $\mathrm{NN}$ affecting the interferometer test masses directly [20]. The miniSOGRO signals could then be used to clean out the NN from the interfero meter.

I acknowledge useful discussions with Peter Shawhan, Rai Weiss, Rana Adhikari, Yanbei Chen, Brian Lantz, Hyung Mok Lee, Ettore Majorana, Runqiu Liu, Gungwon Kang, John Oh, Chunglee Kim and Yongho Lee. I have benefited from collaborations with Jan Harms, Vol Moody, Cornelius Griggs, Xing Bian and Krishna Venkateswara, and am indebted to Ron Norton for laboriously carrying out FEM analysis for numerous platform designs and performing many other calculations. This work was partly supported by NSF grant PHY1105030 and NASA grant NNX14AI43G.

\section{References}

1. B. P. Abbott et al. (LIGO Scientific Collaboration and Virgo Collaboration), Phys. Rev. Lett. 116, 061102 (2016)

2. B. P. Abbott et al. (LIGO Scientific Collaboration and Virgo Collaboration), Phys. Rev. Lett. 116, 241103 (2016)

3. B. P. Abbott et al. (LIGO Scientific and Virgo Collaboration), Phys. Rev. Lett. 118, 221101 (2017)

4. http://www.aei.mpg.de/2068798/lis aselection

5. M. Ando et al., Class. Quantum Grav. 27, 084010 (2010)

6. J. Harms et al., Phys. Rev. D 88, 122003 (2013)

7. H. J. Paik et al., Clas s. Quantum Grav. 33, 075003 (2016)

8. R. V. Wagoner, C. M. Will and H. J. Paik, Phys. Rev. D 19, 2325 (1979)

9. C. E. Griggs et al., Proc. 46th Lunar and Planetary Conference (Houston, Texas, 2015), pp. 1735-1736 http://www.hou.usra.edu/meetings/lpsc2015/pdf/1735.pdf

10. C. Cinquegrana et al., Phys. Rev. D 48, 448 (1993)

11. P. R. Sauls on, Phys. Rev. D 42, 2437 (1990)

12. P. Falferi et al., Appl. Phys. Lett. 93, 172506 (2008)

13. J. R. Bowman, G. E. Baker and M. Bahavar, Geophys. Res. Lett. 32, L09803 (2005)

14. M. G. Beker et al., J. Phys .: Conf. Ser. 363, 012004 (2012)

15. M. V. Moody, H. J. Paik and E. R. Canavan, Rev. Sci. Instrum. 73, 3957 (2002)

16. J. Harms and H. J. Paik, Phys. Rev. D 92, 022001 (2015)

17. J. Harms et al., Geophys. J. Int. 201, 1416(2015)

18. LIGO Science Collaboration, Instrument s cience white paper LIGO DCC T1400316v4 (2015) https://dcc.ligo.org/public/0113/T1400316/004/T1400316-v5.pdf

19. ET Science Team, Einstein gravitational wave telescope conceptual design study (2011) https://tds.ego-gw.it/itf/tds/cod ET-0106C-10

20. H. J. Paik and J. Harms, Proc. 11th Edoardo Amaldi Conf. on Gravitational Waves (Gwangju, Korea, 2015) 